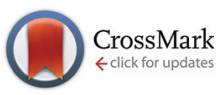

Cite this: Org. Biomol. Chem., 2016, 14, 9736

Received 30th July 2016,

Accepted 27th September 2016

DOI: $10.1039 / c 6 o b 01642 a$

www.rsc.org/obc

\section{Disrupting the PCSK9/LDLR protein-protein interaction by an imidazole-based minimalist peptidomimetic $\uparrow$}

\author{
Mattia Stucchi, ${ }^{a} a, b$ Giovanni Grazioso, ${ }^{c}$ Carmen Lammi, *c Silvia Manara, ${ }^{c}$ \\ Chiara Zanoni, ${ }^{C}$ Anna Arnoldi, ${ }^{C}$ Giordano Lesma ${ }^{a}$ and Alessandra Silvani ${ }^{a}$
}

Herein we report on the multicomponent synthesis of a novel imidazole-based compound, able to act efficiently as a minimalist $\beta$-strand mimic. Biological evaluation proved its ability to impair the LDLR-PCSK9 protein-protein interaction, disclosing it as the first small molecule exerting a PCSK9-mediated hypocholesterolemic effect.

\section{Introduction}

Protein-protein interactions (PPIs) determine the biological role of the relative proteins. Only in the last decade they have begun to be considered as viable targets for therapeutic intervention, dysregulation of PPIs being the cause of many diseases, such as cancer, diabetes, neurodegeneration and HIV. ${ }^{1}$ Quite recently, the PPI between the proprotein convertase subtilisin/kexin type 9 (PCSK9) and the hepatic low density lipoprotein receptor (LDLR) has attracted the attention of the scientific community. ${ }^{2}$ More in depth, PCSK9 is expressed primarily in liver, kidney, and intestine. ${ }^{3}$ It binds to LDLR, promoting its degradation, ${ }^{4,5}$ which results in an increased level of plasmatic low-density lipoprotein. A high concentration of plasma LDL cholesterol is a major cause of atherosclerosis, which subsequently promotes the development of cardiovascular diseases. ${ }^{6,7}$ For this reason, over the past few years PCSK9 has become a sure and potent target for the treatment of hypercholesterolemia. ${ }^{2}$ Moreover, as most of statin drugs' patents have expired recently, numerous pharmaceutical industries are devoting efforts to develop new mole-

\footnotetext{
${ }^{a}$ Dipartimento di Chimica, Università degli Studi di Milano, Via Golgi 19, 20133 Milano, Italy. E-mail: mattia.stucchi@unimi.it

${ }^{b}$ Dipartimento di Scienze della Vita, Università di Modena e Reg-gio Emilia, via G. Campi 103, 41125 Modena, Italy

${ }^{c}$ Dipartimento di Scienze Farmaceutiche, Università degli Studi di Milano,

Via L. Mangiagalli 25, 20133 Milano, Italy. E-mail: giovanni.grazioso@unimi.it, carmen.lammi@unimi.it

$\dagger$ Electronic supplementary information (ESI) available: Synthetic, computational and biological experimental procedures, and spectroscopic data for all synthesized compounds, Fig. S1 and S2, Table S1. See DOI: 10.1039/c6ob01642a
}

cules that can be used in patients with hypercholesterolemia, in combination with, or as alternatives to, statins. On the other hand, the only PCSK9 inhibitors approved for clinical use are, at the moment, expensive monoclonal antibodies, such as evolocumab and alirocumab. ${ }^{8}$

In the search for alternatives to the current therapy, the design of small molecules able to efficiently impair the PCSK9/ LDLR PPI may become an attractive approach. ${ }^{2}$ In general, it is well known that the use of small peptidomimetics of the main recognition motifs is a promising way to disrupt PPIs. ${ }^{9}$ In this context, while $\alpha$-helix ${ }^{10}$ and $\beta$-turn ${ }^{11}$ domains have been extensively investigated, the $\beta$-strand motif has received less attention, ${ }^{12}$ even though some $\beta$-strand peptidomimetics have been successfully employed to inhibit enzymes' activity, in order to treat different diseases, ranging from cancer and AIDS to anthrax and Alzheimer's. ${ }^{13}$

Concerning PCSK9/LDLR PPI, the X-ray crystal structure ${ }^{14}$ clearly reports evidence of a $\beta$-strand-mediated interaction. In particular, PCSK9 comes into contact with the EGF-A domain of LDLR by means of a $\beta$-sheet shaped by the residues C378F379-V380-S381 of PCSK9 and V328-C329-N330-D331 (VCND) of LDLR (Fig. 1).

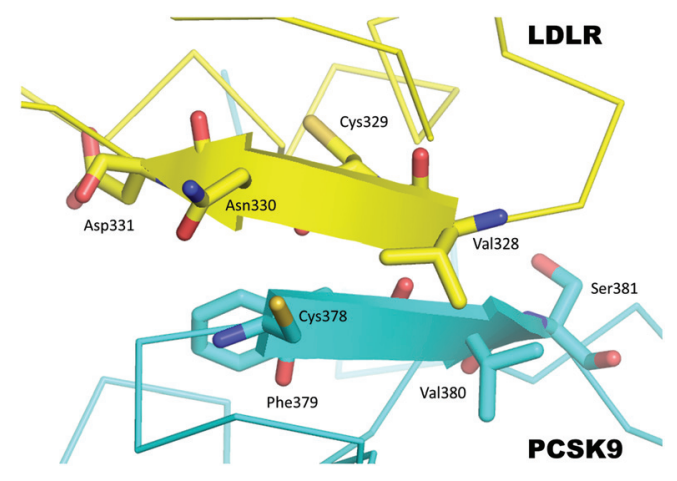

Fig. 1 Schematic representation of the PCSK9/LDLR PPI, as found in the $\mathrm{X}$-ray structure of the complex. 
Going on with our studies on PCSK9/LDLR PPI, we reasoned that small, non-peptidic $\beta$-strand foldamers, resembling or not the VCND sequence, could be able to positively interfere with the PCSK9/LDLR reciprocal interaction.

Initial efforts towards the synthesis of a non-peptidic $\beta$-strand foldamer could be traced back to the work of Hirschmann and Smith in 1992. ${ }^{15}$ Their innovative idea was to replace the peptidic backbone with a heterocyclic scaffold, namely a polypyrrolinone, able to mimic native $\beta$-strands with regard to both the side chain orientations and the inter-strand $\mathrm{H}$-bond donating capabilities. Later, various non-peptidic $\beta$-strand mimics have been developed on the basis of Hirschmann and Smith's concept. ${ }^{16}$ A milestone in the peptidomimetic chemistry was then posed by Burgess and coworkers in $2011,{ }^{17}$ with the definition of minimalist mimics, as frameworks in which only selected side chains of the original peptide chain are present. In this context, Hamilton and
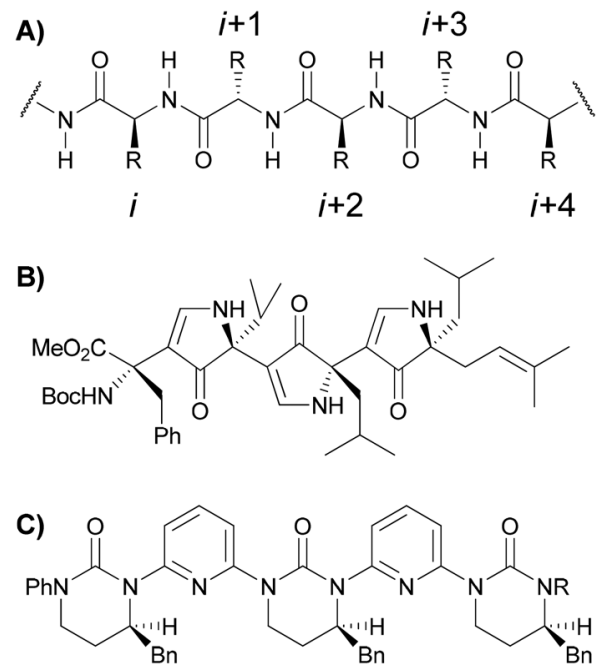

Fig. 2 (A) Native $\beta$-strand. (B) Hirschmann and Smith's $\beta$-strand foldamer. ${ }^{15}$ (C) Hamilton's $\beta$-strand minimalist mimic. ${ }^{18}$ co-workers ${ }^{18}$ recently reported non-peptidic minimalist frameworks, able to mimic the $i, i+2$ and $i+4$ residues of a $\beta$-strand motif, stabilizing their conformations either by intramolecular hydrogen bonds or by the dipolar repulsion effect (Fig. 2).

Relying on our experience with the synthesis of minimalist peptidomimetics, ${ }^{19}$ we conceived a multicomponent reaction (MCR)-based approach to a novel class of potential $\beta$-strand mimics, which are characterized by the presence of multiple C2-C5'-linked imidazole rings, spanning N-R substituents in place of amino acid side chains (Scheme 1). The synthetic strategy is based on the van Leusen three-component reac$\operatorname{tion}^{20}$ (vL-3CR)/C2-formylation iterative protocol. The vL-3CR is able to generate 1,4,5-trisubstituted imidazoles in a single step, by a simple base-induced condensation between an aldehyde, a primary amine and tosylmethyl isocyanide (TosMIC). ${ }^{20}$

As a proof of concept of the goodness of our approach, herein we report the synthesis of the $N$-methyl tetraimidazole derivative 7 (Scheme 2), demonstrating its ability to mimic a $\beta$-strand motif, by means of NMR, computational studies and biological evaluation of PCSK9-LDLR PPI.

\section{Results and discussion}

Although diverse reaction conditions have been reported for the $\mathrm{vL}-3 \mathrm{CR},{ }^{20}$ the combination of potassium carbonate as a base and a highly polar solvent such as methanol, ethanol or dimethylformamide (DMF) usually provides best results. ${ }^{21}$ Therefore, we selected high-boiling DMF as the solvent, and introduced a pre-condensation time of two hours, in order to allow the in situ formation of the corresponding imine, starting from the simple and easy-to-handle benzaldehyde and aqueous methylamine.

The subsequent addition of potassium carbonate and TosMIC, followed by a reaction time of 24 hours at $50{ }^{\circ} \mathrm{C}$, smoothly afforded the desired imidazole derivative $\mathbf{1}$ in high yield. Starting from 1, we applied iteratively the vL-3CR conditions on intermediate formyl derivatives 2,4 and 6 . In this

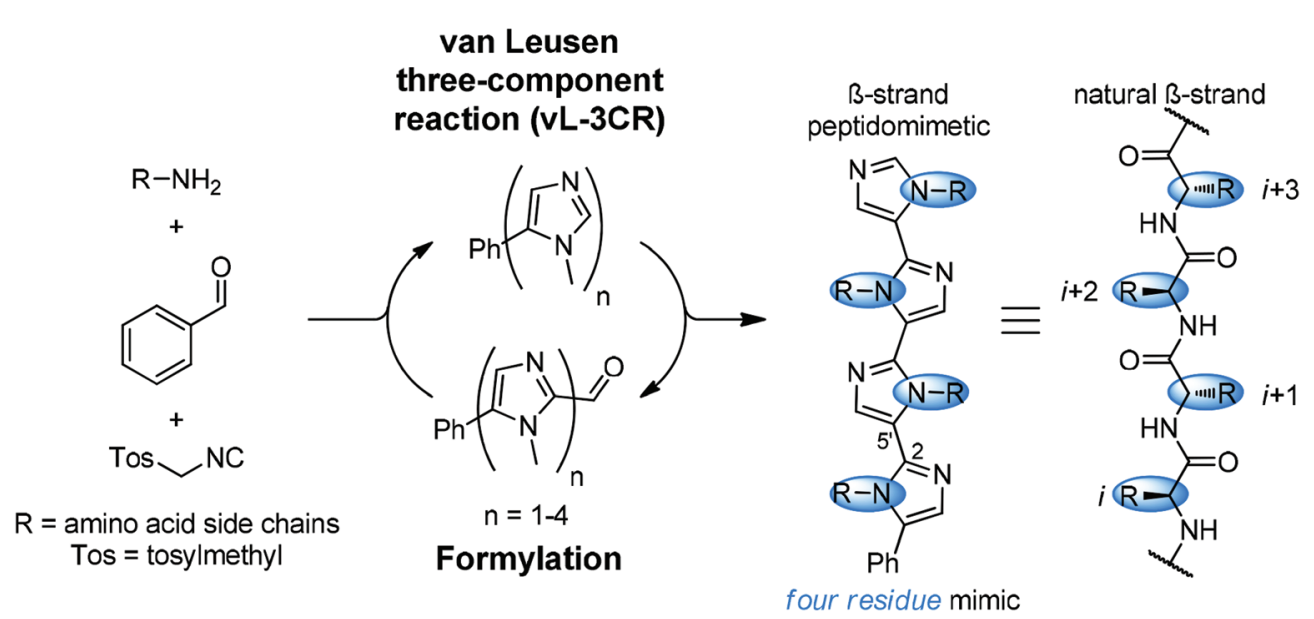

Scheme 1 A non-peptidic, multicomponent approach to the synthesis of $\beta$-strand mimics. 


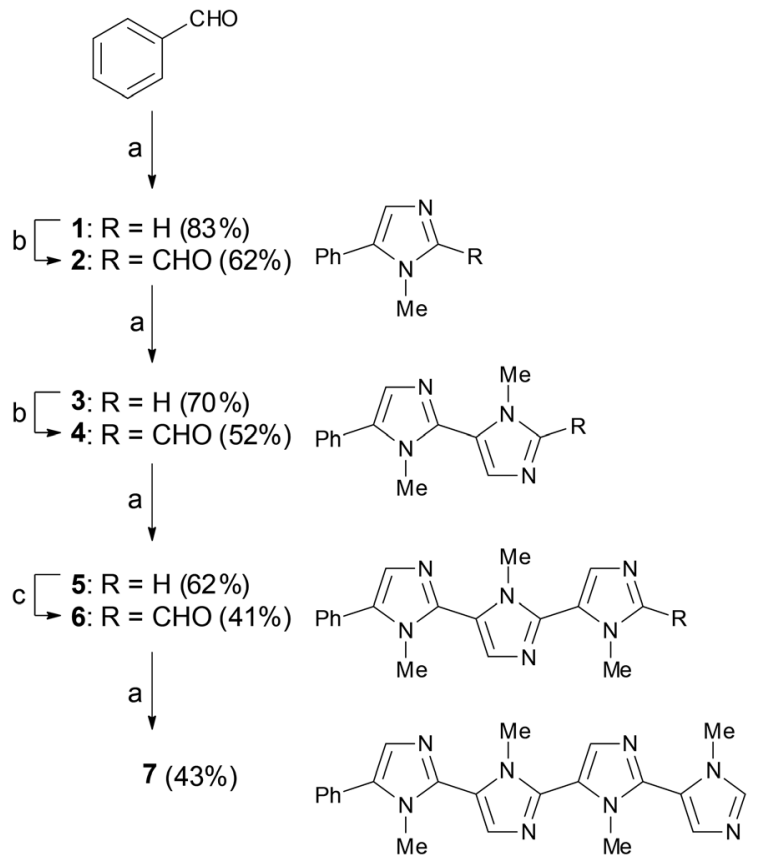

Scheme 2 Synthesis of tetraimidazole 7. Reaction conditions: (a) $\mathrm{MeNH}_{2}\left(40\right.$ wt\% $\mathrm{H}_{2} \mathrm{O}, 2$ eq.), DMF (1-0.25 M), $2 \mathrm{~h}$, rt; then $\mathrm{K}_{2} \mathrm{CO}_{3}(1.5$ eq.), TosMIC (1.2 eq.), $24 \mathrm{~h}, 50^{\circ} \mathrm{C}$; (b) BuLi (1.5 eq.), THF (0.5-0.25 M), $2 \mathrm{~h},-78^{\circ} \mathrm{C}$, then DMF ( 2 eq.), -78 to $\mathrm{rt}, 24 \mathrm{~h}$; (c) BuLi (1.5 eq.), TMEDA ( 2 eq.), THF $(0.05 \mathrm{M}), 2 \mathrm{~h},-78^{\circ} \mathrm{C}$; then DMF (2 eq.), -78 to rt, $24 \mathrm{~h}$.

way, we achieved bi- and tri-imidazoles $\mathbf{3}$ and $\mathbf{5}$ and finally the desired four residue mimic 7. Intermediates 2,4 and 6 could be obtained by classical formylation conditions, using $n$-butyllithium and dimethylformamide at low temperature. In particular, starting from $\mathbf{1}$ or $\mathbf{3}$, the corresponding aldehydes 2 and $\mathbf{4}$ were smoothly achieved in moderate to good yields. Otherwise, under the same conditions, no appreciable formation of the formylated triimidazole 6 could be observed, due to the low solubility of the lithiated derivative of compound 5. However, by adding TMEDA and lowering the reaction concentration, we were able to obtain compound $\mathbf{6}$ in moderate yield.

With tetraimidazole 7 in hand, we investigated its solutionphase conformational behavior through the NOESY NMR experiment in $\mathrm{CDCl}_{3}$ (Fig. 3). Strong correlations between $\mathrm{N}$-Me groups and the aromatic proton of the next ring $(\mathrm{H} 10 \leftrightarrow \mathrm{H} 3, \mathrm{H} 10 \leftrightarrow \mathrm{H} 15, \mathrm{H} 16 \leftrightarrow \mathrm{H} 21, \mathrm{H} 22 \leftrightarrow \mathrm{H} 27$ and $\mathrm{H} 28 \leftrightarrow \mathrm{H} 25$ ) were observed.

On the other hand, not even weak NOE contacts among $N$-Me groups (H10 $\leftrightarrow \mathrm{H} 16, \mathrm{H} 16 \leftrightarrow \mathrm{H} 22$ and $\mathrm{H} 22 \leftrightarrow \mathrm{H} 28)$ could be detected, clearly indicating an $N$-Me alternate, $\beta$-strand-like, conformation for compound 7.

In order to properly assess tetraimidazole 7 as a minimalist $\beta$-strand mimetic, computational studies were performed. Molecular dynamics (MD) simulations with the GB implicit water solvent model were executed by using the AMBER12 package, and GAFF force field. ${ }^{22,23}$ In particular, the computational model of 7 was investigated by minimizing, equili-

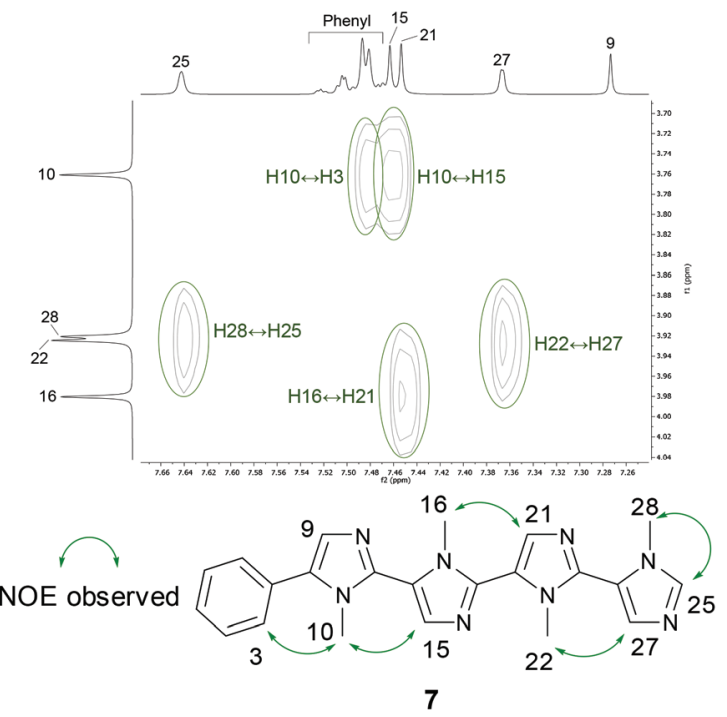

Fig. 3 Selected region of the NOESY NMR $\left(\mathrm{CDCl}_{3}, 400 \mathrm{MHz}, 298 \mathrm{~K}\right)$ spectrum of compound 7, focusing on the cross-peaks between $\mathrm{N}-\mathrm{Me}$ groups and aromatic protons. Green arrows indicate the observed NOE contacts.

brating and heating up the starting conformation to $300 \mathrm{~K}$, $700 \mathrm{~K}$ and $1000 \mathrm{~K}$ for a short period $(2 \mathrm{~ns})$. Then, after an intermediate equilibration step at $700 \mathrm{~K}$, a production run of $20 \mathrm{~ns}$ of MD simulations at $300 \mathrm{~K}$ was accomplished, ${ }^{17}$ acquiring 2800 conformational states. Analyzing the fluctuation of the dihedral angles connecting the imidazole rings, and their distribution percentage ( $\tau 1, \tau 2$ and $\tau 3$ in Fig. $4 \mathrm{~A}$ ), we were able to confirm that compound 7 assumes the conformation suggested by the NOESY experiment, with the most populated values of dihedral angles in the range from $180^{\circ}$ to $150^{\circ}$ and from $-150^{\circ}$ to $-180^{\circ}$ (for additional details see the ESI $\dagger$ ).

To determine the robustness of compound 7 as a $\beta$-strand mimic, the distances between the $N$-Me groups were measured on the ab initio optimized (B3LYP/6-31 g(d)/CPCM-water level of theory) lowest energy conformer. By comparing the found values of 5.5, 7.3 and 11.4 $\AA$ to those reported by Burgess and coworkers ${ }^{17}$ for $\mathrm{C}^{\beta}-\mathrm{C}^{\beta}$ distances in typical secondary structures, we can expect that 7 mimics the parallel $\beta$-strand motif

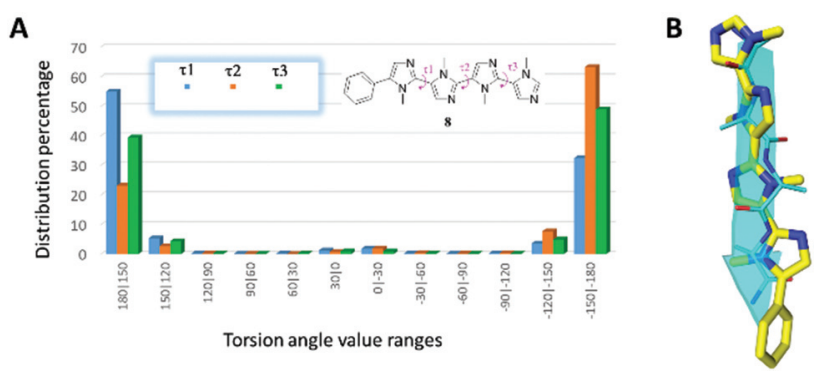

Fig. 4 (A) Torsion angle distributions resulting from the MD simulations of compound 7. (B) Superimposition between 7 (yellow) and a hypothetical protein $\beta$-strand (cyan). 
A

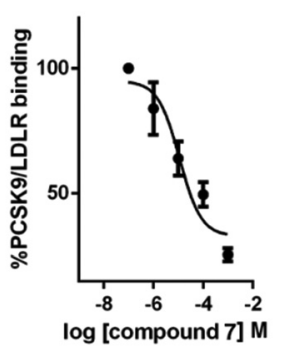

B

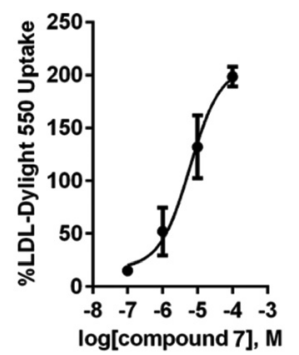

Fig. 5 (A) Inhibitory effects of compound 7 on the PCSK9-LDLR PPI in vitro. Data points represent averages \pm SEM of three independent experiments in triplicate. (B) Effect of compound 7 on the HepG2 ability to uptake LDL from the extracellular environment. Data points represent averages \pm SEM of three independent experiments.

almost perfectly (optimal $i-i+1, i-i+2$ and $i-i+3$ distances are 5.8, 7.1 and $11.1 \AA$ respectively, see Table S1, ESI $\dagger$ ). Moreover, superimposing compound 7 with a hypothetical protein $\beta$-strand motif, we observed very good overlapping (Fig. 4B), with distances between the $N$-Me groups of 7 and the $\mathrm{C}^{\beta}$ atoms of $\beta$-strand side chains lower than $0.5 \AA$ A.

This last observation drove the biological investigation of compound 7's capability to inhibit the therapeutically relevant PPI between PCSK9 and LDLR (Fig. 5). ${ }^{14}$ Remarkably, the in vitro PCSK9-LDLR binding assay showed that compound 7 induces a concentration dependent inhibition of the LDLR binding on PCSK9, with an $\mathrm{IC}_{50}$ value equal to $11.2 \pm 0.2 \mu \mathrm{M}$ (Fig. 5A). In the same experiments, instead, compound 5 gave an $\mathrm{IC}_{50}$ equal to $116.3 \pm 0.16 \mu \mathrm{M}$, indicating that the presence of three imidazole rings in the structure are not enough to efficiently impair the PCSK9-LDLR PPI. For comparison, we refer to two known PCSK9 inhibitors. Both are peptides showing $\mathrm{IC}_{50}$ values in a low micromolar range. The first one, recently reported by us, is a natural decapeptide derived from lupin (P5) and showing an $\mathrm{IC}_{50}$ value equal to $1.6 \mu \mathrm{M} .^{24}$ The other one, singled out by Zhang and coworkers, ${ }^{25}$ is Pep2-8 (13 amino acids) displaying an $\mathrm{IC}_{50}$ value of $0.8 \mu \mathrm{M}$. The disclosed micro-molar $\mathrm{IC}_{50}$ of compound 7 , along with its peptidomimetic nature, is in our opinion particularly relevant and can pave the way for an original, non-peptidic approach to PCSK9 inhibition. With this in mind, we carried out deeper investigation into the activity of compound 7 . Thus, the change of the functional capability of HepG2 cells, previously treated with this compound, to uptake extracellular LDL was investigated, by performing fluorescent-LDL uptake experiments. We could observe that the ability of compound 7 to impair the PCSK9-LDLR binding, stabilizing the active LDLR on the cell membrane, leads to an improved ability of hepatic cells to uptake extracellular LDL, with a final hypocholesterolemic effect (Fig. 5B). This evidence indicates that compound 7 does not directly bind the LDLR, localized on the cellular membranes; otherwise the improved capacity of HepG2 cells to absorb extracellular fluorescent LDL would have been severely impaired. MTT experiments have also been per-

formed, in order to exclude any potential cytotoxicity effect after treatment of HepG2 with 1, 10, 50, and $100 \mu \mathrm{M}$ of compound 7 for $24 \mathrm{~h}$. As clearly shown by the attained results (Fig. S1, see the ESI $\dagger$ ), compound 7 is safe for HepG2 cells.

In conclusion, we have developed a novel synthetic route to a C2-C5'-linked tetraimidazole scaffold, by means of an iterative vL-3CR/C2-formylation protocol. Its solution-phase conformational behaviour was investigated through NOESY, NMR and computational studies, demonstrating its ability to mimic the $i, i+1, i+2$ and $i+3$ amino acid residues of a $\beta$-strand motif. This evidence led us to test the capability of this compound to effectively disrupt a $\beta$-strand-mediated PPI. With this aim, the therapeutically relevant PCSK9-LDLR PPI was chosen as the biological target. The attained results confirmed that the tetraimidazole scaffold is able to impair the PCSK9-LDLR reciprocal interaction, with an $\mathrm{IC}_{50}$ value equal to $11.2 \mu \mathrm{M}$. Also the LDL-uptake is increased $\left(\mathrm{EC}_{50}=6.04 \mu \mathrm{M}\right)$, while MTT assays assured that such a compound is also safe on liver HepG2 cells.

Undoubtedly, compound 7 constitutes a versatile scaffold for a generation of new molecular entities capable of potentially mimicking any $\beta$-strand motif. Furthermore, since there are no reports yet in the literature on small molecules able to inhibit PCSK9, we are also confident that compound 7 will constitute a lead compound for the rational design of a new class of PCSK9 inhibitors. Further work is underway in order to better refine the chemical framework, in particular, through substitution of $N$-Me groups with different residues, more strictly resembling the actual LDLR $\beta$-strand side chains.

We are grateful for financial support from the University of Milan, Grant 2015, Line 2 - Action A (PI GG).

\section{Notes and references}

1 (a) L. Garner and K. D. Janda, Curr. Top. Med. Chem., 2011, 11, 258; (b) B. O. Villoutreix, M. A. Kuenemann, J. Poyet, H. Bruzzoni-Giovanelli, C. Labbé, D. Lagorce, O. Sperandio and M. A. Miteva, Mol. Inf., 2014, 33, 414; (c) T. L. Nero, C. J. Morton, J. K. Holien, J. Wielens and M. W. Parker, Nat. Rev. Cancer, 2014, 14, 248.

2 N. Bergeron, B. A. P. Phan, Y. Ding, A. Fong and R. M. Krauss, Circulation, 2015, 132, 1648.

3 N. G. Seidah and A. Prat, Nat. Rev. Drug Discovery, 2012, 11, 367.

4 D. W. Zhang, T. A. Lagace, R. Garuti, Z. Zhao, M. McDonald, J. D. Horton, J. C. Cohen and H. H. Hobbs, J. Biol. Chem., 2007, 282, 18602.

5 B. Gencer, G. Lambert and F. Mach, Swiss Med. Wkly., 2015, 145, w14094.

6 J. G. Canto and A. E. Iskandrian, JAMA, J. Am. Med. Assoc., 2003, 290, 947.

7 E. G. Nabel, N. Engl. J. Med., 2003, 349, 60.

8 B. M. Everett, R. J. Smith and W. R. Hiatt, N. Engl. J. Med., 2015, 373, 1588. 
9 (a) S. Jones and J. M. Thornton, Proc. Natl. Acad. Sci. U. S. A., 1996, 93, 13; (b) I. S. Moreira, P. A. Fernandes and M. J. Ramos, Proteins: Struct., Funct., Bioinf., 2007, 68, 803; (c) L. Garner and K. D. Janda, Curr. Top. Med. Chem., 2011, 11, 258.

10 (a) C. G. Cummings and A. D. Hamilton, Curr. Opin. Chem. Biol., 2010, 14, 341; (b) R. S. Harrison, N. E. Shepherd, H. N. Hoang, G. Ruiz-Gomez, T. A. Hill, R. W. Driver, V. S. Desai, P. R. Young, G. Abbenante and D. P. Fairlie, Proc. Natl. Acad. Sci. U. S. A., 2010, 107, 11686; (c) G. L. Verdine and G. J. Hilinski, Methods Enzymol., 2012, 503, 3; (d) M. D. Boersma, H. S. Haase, K. J. PetersonKaufman, E. F. Lee, O. B. Clarke, P. M. Colman, B. J. Smith, W. S. Horne, W. D. Fairlie and S. H. Gellman, J. Am. Chem. Soc., 2012, 134, 315; (e) V. Azzarito, K. Long, N. S. Murphy and A. J. Wilson, Nat. Chem., 2013, 5, 161.

11 T. A. Hill, N. E. Shepherd, F. Diness and D. P. Fairlie, Angew. Chem., Int. Ed., 2014, 53, 13020.

12 H. Remaut and G. Waksman, Trends Biochem. Sci., 2006, 31, 436.

13 (a) S. Maitra and J. Norwick, The Amide Linkage: Structural Significance in Chemistry, Biochemistry and Material Science, Wiley, New York, 2000, pp. 495-518; (b) W. A. Loughlin, J. D. A. Tyndall, M. P. Glenn, T. A. Hill and D. P. Fairlie, Chem. Rev., 2010, 110, PR32.

14 P. Lo Surdo, M. J. Bottomley, A. Calzetta, E. C. Settembre, A. Cirillo, S. Pandit, Y. G. Ni, B. Hubbard, A. Sitlani and A. Carfi, EMBO Rep., 2011, 12, 1300.

15 A. B. Smith III, T. P. Keenan, R. C. Holcomb, P. A. Sprengeler, M. C. Guzman, J. L. Wood, P. J. Carroll and R. Hirschmann, J. Am. Chem. Soc., 1992, 114, 10672.

16 (a) A. B. Smith III, M. C. Guzman, P. A. Sprengeler, T. P. Keenan, R. C. Holcomb, J. L. Wood, P. J. Carroll and R. Hirschmann, J. Am. Chem. Soc., 1994, 116, 9947; (b) W. A. Loughlin, J. D. A. Tyndall, M. P. Glenn and D. P. Fairlie, Chem. Rev., 2004, 104, 6085; (c) N. G. Angelo and P. S. Arora, J. Am. Chem. Soc., 2005, 127, 17134; (d) S. Chandrasekhar, B. N. Babu, A. Prabhakar, A. Sudhakar, M. S. Reddy, M. U. Kiran and B. Jagadeesh, Chem. Commun., 2006, 1548; (e) M. C. Hammond, B. Z. Harris, W. A. Lim and P. A. Bartlett, Chem. Biol., 2006, 13, 1247; $(f)$ M. C. Hammond and P. A. Bartlett, J. Org. Chem., 2007, 72, 3104; (g) J. L. Watson and E. R. Gillies, J. Org. Chem., 2009, 74, 5953; (h) N. Ross, W. P. Katt and A. D. Hamilton, Philos. Trans. R. Soc. London, Ser. A, 2010, 368, 989; (i) A. Raghuraman, E. Ko, L. M. Perez, T. R. Ioerger and K. Burgess, J. Am. Chem. Soc., 2011, 133, 12350; (j) A. B. Smith III, A. K. Charnley and R. Hirschmann, Acc. Chem. Res., 2011, 44, 180; (k) C. W. Kang, Y. Sun and J. R. Del Valle, Org. Lett., 2012, 14, 6162.

17 (a) E. Ko, J. Liu and K. Burgess, Chem. Soc. Rev., 2011, 40, 4411; (b) E. Ko, J. Liu, L. M. Perez, G. Lu, A. Schaefer and K. Burgess, J. Am. Chem. Soc., 2011, 133, 462.

18 (a) P. N. Wyrembak and A. D. Hamilton, J. Am. Chem. Soc., 2009, 131, 4566; (b) A. G. Jamieson, D. Russell and A. D. Hamilton, Chem. Commun., 2012, 48, 3709; (c) C. L. Sutherell, S. Thompson, R. T. W. Scott and A. D. Hamilton, Chem. Commun., 2012, 48, 9834; (d) E. A. German, J. E. Ross, P. C. Knipe, M. F. Don, S. Thompson and A. D. Hamilton, Angew. Chem., Int. Ed., 2015, 54, 2649; (e) T. Yamashita, P. C. Knipe, N. Busschaert, S. Thompson and A. D. Hamilton, Chem. Eur. J., 2015, 21, 14699.

19 M. Stucchi, S. Cairati, R. Cetin-Atalay, M. S. Christodoulou, G. Grazioso, G. Pescitelli, A. Silvani, D. C. Yildirim and G. Lesma, Org. Biomol. Chem., 2015, 13, 4993.

20 (a) A. M. van Leusen and J. Strating, Q. Rep. Sulfur Chem., 1970, 5, 67; (b) A. M. van Leusen, J. Wildeman and O. Oldenziel, J. Org. Chem., 1977, 42, 1977; (c) D. van Leusen and A. M. van Leusen, Org. React., 2003, 57, 419.

21 (a) H. Che, T. N. Tuyen, H. P. Kim and H. Park, Bioorg. Med. Chem. Lett., 2010, 20, 4035; (b) C. Lamberth, R. Dumeunier, S. Trah, S. Wendeborn, J. Godwin, P. Schneiter and A. Corran, Bioorg. Med. Chem., 2013, 21, 127.

22 D. A. Case, T. A. Darden, T. E. Cheatham III, C. L. Simmerling, J. Wang, R. E. Duke, R. Luo, R. C. Walker, W. Zhang, K. M. Merz, B. Roberts, S. Hayik, A. Roitberg, G. Seabra, J. Swails, A. W. Götz, I. Kolossváry, K. F. Wong, F. Paesani, J. Vanicek, R. M. Wolf, J. Liu, X. Wu, S. R. Brozell, T. Steinbrecher, H. Gohlke, Q. Cai, X. Ye, J. Wang, M.-J. Hsieh, G. Cui, D. R. Roe, D. H. Mathews, M. G. Seetin, R. Salomon-Ferrer, C. Sagui, V. Babin, T. Luchko, S. Gusarov, A. Kovalenko and P. A. Kollman, AMBER 12, University of California, San Francisco, 2012.

23 J. Wang, R. M. Wolf, J. W. Caldwell, P. A. Kollman and D. A. Case, J. Comput. Chem., 2004, 25, 1157.

24 C. Lammi, C. Zanoni, G. Aiello, A. Arnoldi and G. Grazioso, Sci. Rep., 2016, 6, 29931.

25 Y. Zhang, C. Eigenbrot, L. Zhou, S. Shia, W. Li, C. Quan, J. Tom, P. Moran, P. Di Lello, N. J. Skelton, M. KongBeltran, A. Peterson and D. Kirchhofer, J. Biol. Chem., 2014, 289, 942. 